\title{
The Impact of Transmission Power on the Performance of MANET Routing Protocols
}

\author{
V.Lalitha ${ }^{1 *}$, Dr.R.S.Rajesh ${ }^{2}$ \\ ${ }^{1 *}$ Dept. of Computer Science, Govt. Arts College, Pudukkottai (TN), India \\ ${ }^{2}$ Dept. of Computer Science\&Engineering, M.S.University, Tirunelveli (TN), India
}

\begin{abstract}
A Mobile Ad hoc Network is an infrastructure-less multi-hop wireless network. A node communicates directly with other mobile nodes within the wireless range and indirectly with nodes that are not within the wireless range of each other. Transmission power of the nodes plays an important role here. In a multi hop communication scenario, it is possible to minimize the total energy for reaching a higher distance by repeated low power transmissions. The objective of this work is to study the behavior and performance of the three frequently used MANET routing protocols with respect to different transmission power of individual nodes of the network. We have selected Destination Sequenced Distance Vector (DSDV) Routing Protocol, Ad Hoc On-Demand Distance Vector (AODV) Routing Protocol and Dynamic Source Routing (DSR) Protocol for this study. We analyzed the positive and negative impact of increase in transmission power of individual nodes on the performance of these routing protocols. The arrived results are more significant and can be used in the design of new power aware routing protocols.
\end{abstract}

Key Words: AODV, DSDV, DSR, MANET, transmission power, Power Aware Routing Protocols.

\section{INTRODUCTION}

A MANET is viewed as a collection of mobile nodes that are equipped with wireless receivers and transmitters and are free to move around. At any given time, the status of the communication links between the nodes depends on various factors, which include their positions, their velocities, the transmission power levels, and antenna patterns. Moreover, the topology of the network changes in time since the nodes move freely relative to each other. One of the challenging issues is the node mobility that causes frequent link failures which result in serious performance degradation. The links in MANET are mostly unidirectional and the node transmission power is affected by interferences. So the nodes use different transmission powers.

Suman Banerjee and Archan Misra [13] have analyzed multi-hop networks in variable-power scenarios, where the nodes can dynamically vary their transmitter power levels. In such cases, greater energyefficiency can be obtained if the nodes choose the transmission power depending on the distance between the transmitter and receiver nodes.

The transmission range of the node is a very important aspect which will have more influence on the network connectivity. The nodes with sufficiently higher transmission range can maintain connectivity even at higher mobility. In a multi hop communication scenario, there may be frequent link failures because of rapid change in topology due to node mobility. If we increase the transmission range of power to increase the one hop distance of the nodes, then it will certainly avoid link failures due to mobility to some extent. In this study, we will analyze the impact of change in transmission range or power on the performance of MANET routing algorithms.

\section{TRANSMISSION POWER MODEL AND ENERGY CONSERVATION}

The most common propagation models adopted in MANET simulations to predict the received signal power of each packet are

* free space model

* two-ray ground reflection model and

* the shadowing model

\subsection{Free Space Model}

The free space model [1 2] assumes the ideal propagation condition of clear line-of-sight path between the transmitter and receiver. The equation for this model by Friis [4] is given by

$$
P_{r}(d)=\frac{P_{t} G_{t} G_{r} \lambda^{2}}{(4 \pi)^{2} d^{2} L}
$$

For Omni-directional antenna $\mathrm{G}_{\mathrm{t}}=\mathrm{Gr}_{\mathrm{r}}=1$ and $\mathrm{L}$, is usually set to1. 


\subsection{Two-ray ground reflection model}

The two-ray ground reflection model [1 2] also considers the ground refection path besides the lineof-sight propagation. For longer distance more accurate prediction is obtained by using this model. The equation is given by

$$
P_{r}(d)=\frac{P_{t} G_{t} G_{r} h_{t}^{2} h_{r}^{2}}{d^{4} L}
$$

Two-ray model is used for 1 onger distan ces. Usually, if $\mathrm{d}$ is smaller than the cross-over distance $\left(\mathrm{d}_{\mathrm{C}}\right)$

$d_{c}=4 \pi h_{t} h_{r} / \lambda$

then free space model Eq. (1) is used otherwise Eq. (2) is used.

In the above equations, the symbols means the following

$\operatorname{Pr} \quad$ is the Received Signal Power

$\mathrm{Pt}_{\mathrm{t}} \quad$ is the Transmitted Signal Power

$\mathrm{G}_{\mathrm{t}} \quad$ is the Transmission Gain of Antenna

$\mathrm{G}_{\mathrm{r}} \quad$ is the Receiver Gain of Antenna

$\mathrm{L} \quad$ is the System Loss

$\lambda \quad$ is the Wavelength

D is the Distance between the Transmitter and Receiver

$\mathrm{h}_{\mathrm{r}} \quad$ is the Height of antenna for receiver

$h_{t} \quad$ is the Height of antenna for transmitter

\subsection{Shadowing Model}

The space model and the two-ray model both represent the communication range as the ideal circle. They do not include the multi-path propagation effects and the fading effects that impart random behavior to the received power at a certain distance. The shadowing model [13] captures these effects into consideration.

\section{TRANSMISSION-DISTANCE CALCULATION}

There are too many factors that affect the transmission distance, particularly the combination of transmission power and antenna gain.

The theoretical transmission distance that can be achieved between two wireless devices can be calculated from a few key specifications.
* Tx power
* Data rate
- Rx sensitivity
* Antenna Gain
* Frequency

The transmission power of the device $\mathrm{T} x$ is measured in $\mathrm{dBm}$. The data rate is the number of bits processed per unit time. The receiver sensitivity Rx is measured in $\mathrm{dBm}$. The gain for an antenna is measured in $\mathrm{dBi}$ and it indicates how much the signal is boosted by the antenna. The frequency is typically measured in $\mathrm{MHz}$ or $\mathrm{GHz}$ and indicates which electromagnetic band is used for wireless communication

\subsection{Calculation of Transmission Power with respect to Range}

The following table shows the coverage distance and the required transmission power for that distance. We have set the Tx power of the node based on this relationship. In the following table, the transmission power is calculated based on the needed coverage distance. If the distance $d$ is smaller than the cross over distance $d_{c}$ (Eq. 3), then free space model (Eq. 1) is used otherwise the two-ray ground model (Eq. 2) is used.

\begin{tabular}{|c|c|}
\hline \multicolumn{2}{|c|}{ Table 1.The. Tx Power and Range of Transmission } \\
\hline Distance & Transmission Power Needed \\
\hline 100 & 0.007214 \\
150 & 0.036520 \\
200 & 0.115421 \\
250 & 0.281790 \\
300 & 0.584320 \\
350 & 1.082525 \\
450 & 1.846740 \\
500 & 2.958120 \\
\hline
\end{tabular}




\section{SIMULATION ANALYSIS}

The ns2 [3] simulation tool is selected to study all the behavior of MANET routing protocol. CMU's wireless extension to ns-2 provides the implementation of the DSR, AODV and DSDV routing protocols.

\subsection{MANET Routing Protocols Destination Sequenced Distance Vector (DSDV) Routing Protocol}

DSDV [5 10] is a table driven routing protocol which is based on the Bellman-Ford routing algorithm [6]. DSDV is suitable when almost all nodes are mutually involved in communication with almost all other nodes and in which the mobility factor is medium.

\section{Ad Hoc on-Demand Distance Vector (AODV) Routing Protocol}

AODV [7 8] is a reactive routing protocol and the routes are calculated only when needed. It maintains these routes as long as they are needed by the source. It uses trees to connect multicast group members. It is selfstarting, loop free and highly scalable. It supports both unicast and multicast routing.

\section{Dynamic Source Routing (DSR) Protocol}

DSR [9] gives rise to a completely self-organizing and self-configuring network without the need for any existing network infrastructure or administration. It uses Route Discovery and Route Maintenance mechanisms to discover and maintain source routes to arbitrary destinations.

\subsection{Random Waypoint Mobility model}

In this model [11], a node picks a destination at random and moves towards it with a speed chosen randomly from a uniform distribution $\left[0, \mathrm{~V}_{\max }\right]$, where $\mathrm{V}_{\max }$ is the highest possible speed for every node. When it arrives at the destination, the node waits for a duration defined by the 'pause time' parameter. After this time has passed, it chooses a new random destination and repeats the process until the end of the simulation.

\subsection{Metrics considered for evaluation}

In order to evaluate the performance of ad hoc network routing protocols, the following metrics were considered:

\section{Packet Delivery Fraction/Ratio (PDF/PDR):}

Packet delivery ratio or fraction is the ratio of the number of packets successfully received by all destinations to the total number of packets injected into the network by all sources.

\section{Routing Load:}

Routing Load means the average number of routing messages generated to each data packet successfully delivered to the destination. It can be measured by the ratio of the number of routing messages propagated by every node in the network and the number of data packets successfully delivered to all destination nodes.

\section{End-to-End Delay:}

The average time interval between the generation of a packet in a source node and the successfully delivery of the packet at the destination node. It counts all possible delays that can occur in the source and all intermediate nodes, including queuing time, packet transmission and propagation, and retransmissions at the MAC layer. The queuing time can be caused by network congestion or unavailability of valid routes. It is measured in milliseconds.

\section{Dropped Packets:}

The dropping of a packet will occur during a collision/error or other failure in routing process. Here the dropped packet count is the number of 'data packets' that are not successfully sent or forwarded to the destination. It is measured in numbers.

\section{Throughput:}

Throughput is the number of bytes or bits arriving at the sink over time. It is generally measured in kilo bits per second or Mega Bits per second.

\section{Energy Consumption:}

The average of the total consumed energy of all the nodes of the network. It is measured in Joules. 
MAC Load:

MAC load means the average number of MAC messages generated to each data packet successfully delivered to the destination. It is the ratio of the number of MAC layer messages propagated by every node in the network and the number of data packets successfully delivered to all destination nodes.

\section{The Overhead:}

We also used the number of generated and forwarded routing messages as separate metric to understand the routing overhead in the scenario.

\subsection{Parameters of the Simulation}

The following table shows some of the important parameters of the simulation. In our simulations, we used very low rate traffic because we may consider this topology as a model of sensor network topology also.

Table 2: Parameters of the Simulation

\begin{tabular}{|c|l|}
\hline Routing Protocols & DSDV, AODV and,DSR \\
Total Nodes & 40 \\
& $100 \mathrm{~m}, 150 \mathrm{~m}, 200 \mathrm{~m}$, \\
Transmission Ranges & $250 \mathrm{~m}, 300 \mathrm{~m}, 350 \mathrm{~m}$, \\
& $400 \mathrm{~m}, 450 \mathrm{~m}, 500 \mathrm{~m} \&$ \\
Rx Power & $550 \mathrm{~m}$ \\
Idle Power & $0.2819 \mathrm{mw}$ \\
Initial Node Energy & $0.14 \mathrm{mw}$ \\
Antenna Model & 1000 Joules \\
Propagation Model & Omni Antenna \\
Mac Type & Two Ray Ground \\
Interface queue type & Do2.11 \\
Topological Area & Drop Tail/PriQueve \\
Node speed & S00 $\mathrm{m}$ X $800 \mathrm{~m}$ \\
Pause Time & $10 \mathrm{~m} / \mathrm{s}$ \\
\hline CBR Traffic Parameters & $10 \mathrm{~s}$ \\
& Interval : 1.0 sec \\
& Packet Size :512 Bytes \\
\hline Transport Protocol & Sources : 10, Sinks : 10 \\
\hline & UDP \\
\hline
\end{tabular}

\section{RESULTS}

The performance of the routing protocols with respect to different transmission range of the nodes are shown graphically. The following graph shows the performance of the routing protocols in terms of overhead. As shown in Fig. 1 the overhead in the case of DSDV is very high. But the overhead seems to be reducing along with the increase in transmission range. DSR performed good in terms of overhead.

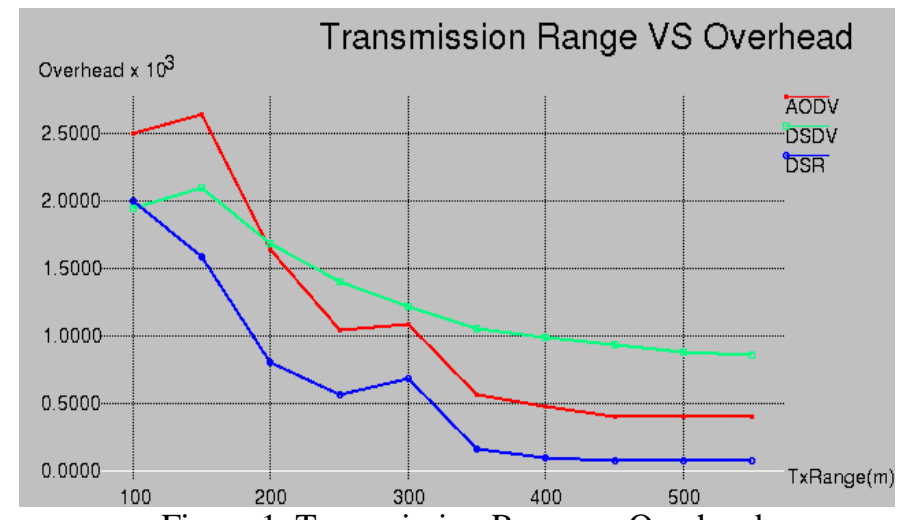

Figure 1, Transmission Range vs Overhead

The following graph shows the performance of the protocols in terms of average consumed energy. As shown in the figure 2, the DSDVprotocol consume much energy than the other two protocols. Further, the average energy consumption seems to be incerasing along with the increase in transmission range. Between the range $250 \mathrm{~m}$ to $400 \mathrm{~m}$, the algorithms provided optimum level of performance. DSR performed good in terms of consumed energy. 


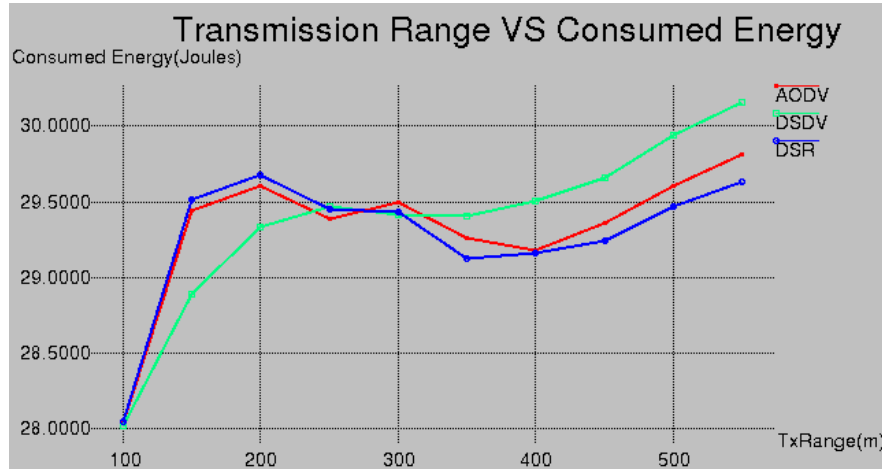

Figure 2, Transmission Range vs Average Consumed Energy

The following graph shows the performance of the protocols in terms of end to end delay. As shown in the figure 3, the end do end delay below the transmission range of 200 was high. During low transmission power, the routing will happen through multiple hops and hence increase the end to end delay. But beyond the $200 \mathrm{~m}$ transmission range, the end to end delay was very much reduced to a very lower level. DSDV provided minimum End to End Delay.

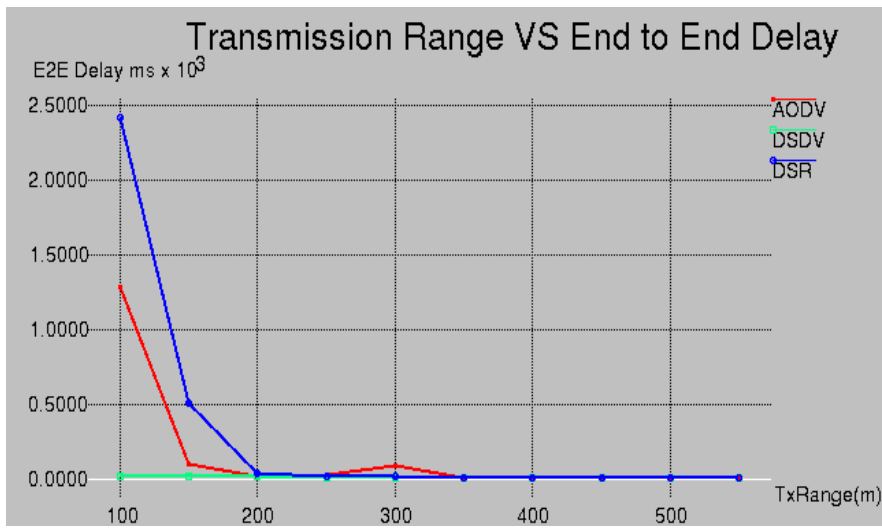

Figure 3, Transmission Range vs End to End Delay

The following graph shows the performance of the protocols in terms of dropped packets. As shown in the figure 4, the protocols actually drop packet only during using low transmission power. But beyond the $400 \mathrm{~m}$ transmission range, the packet dropping was almost zero. AODV is dropping lesser packets if the Tx Range is below $350 \mathrm{~m}$.

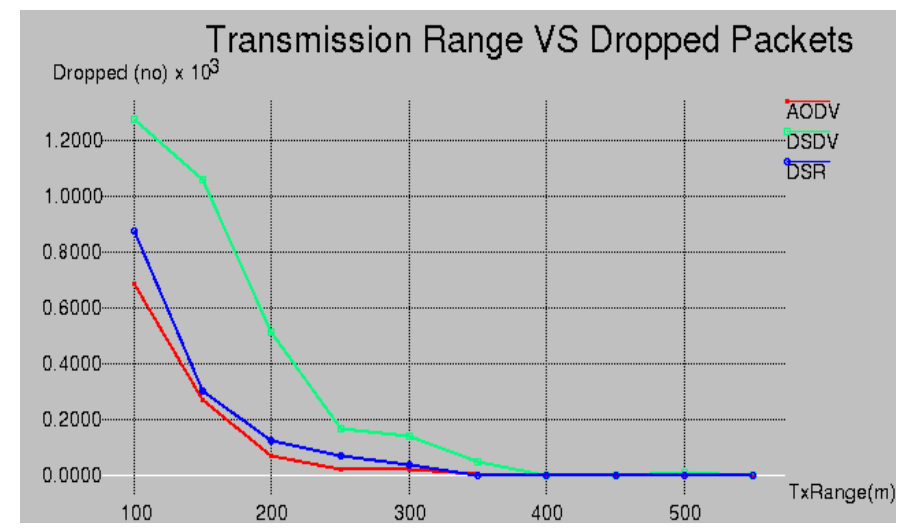

Figure 4, Transmission Range vs Dropped Packets

The following graph shows the performance of the protocols in terms of routing load. As shown in the figure 5, the protocols actually experience much routing load only during using low transmission power. But beyond the $300 \mathrm{~m}$ transmission range, the routing load becomes minimum and almost constant. DSR is dropping lesser packets if the Tx Range is below $350 \mathrm{~m}$. 


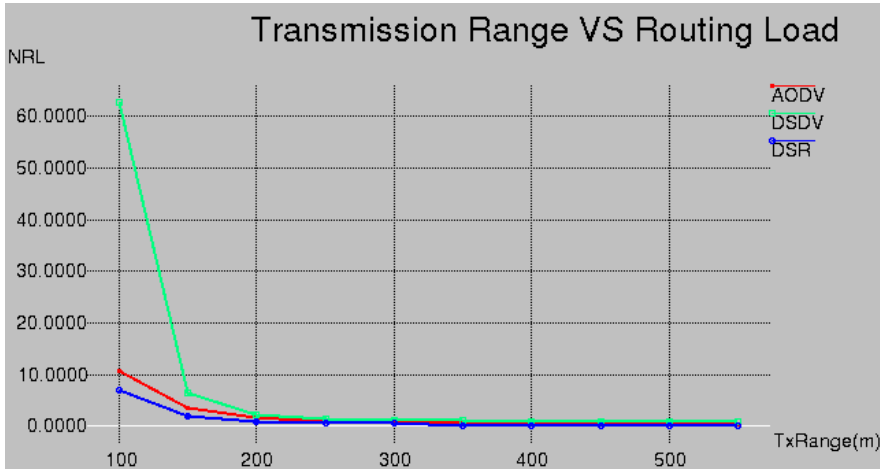

Figure 5, Transmission Range vs Routing Load

The following graph shows the performance of the protocols in terms of MAC load. As shown in figure 6, the protocols actually expewrience much MAC load only during using low transmission power. But beyond the $300 \mathrm{~m}$ transmission range, the MAC load becomes very minimum and was almost constant. The performance in terms of MAC Load in the case of AODV and DSR are almost equal if the Tx Range is below $350 \mathrm{~m}$.

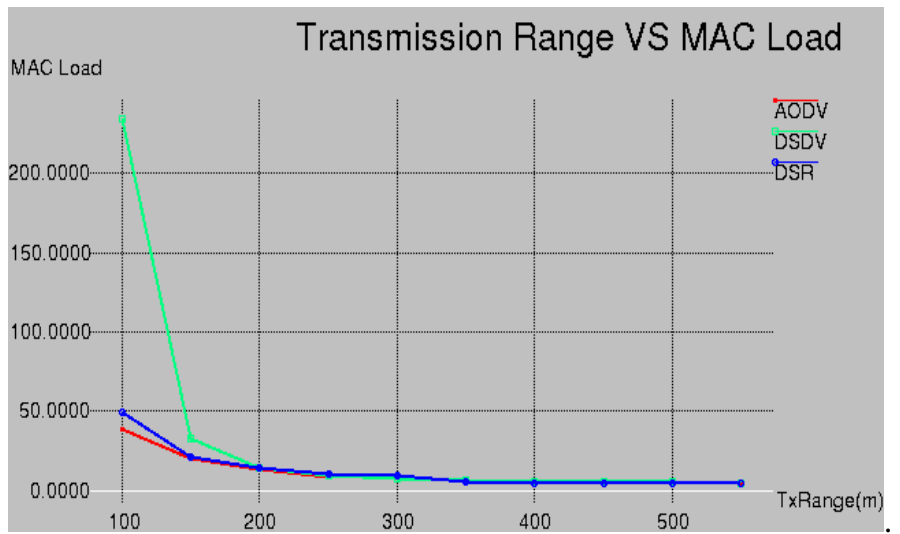

Figure 6, Transmission Range vs MAC Load

The following graph shows the performance of the protocols in terms of PDF. As shown in figure 7, the protocols provided poor packet delivery ratio during low transmission power in the nodes. But beyond the $350 \mathrm{~m}$ of the transmission range, the PDF was ideal and becomes good and almost constant. DSR provided good PDF if the Tx Range is below $350 \mathrm{~m}$.

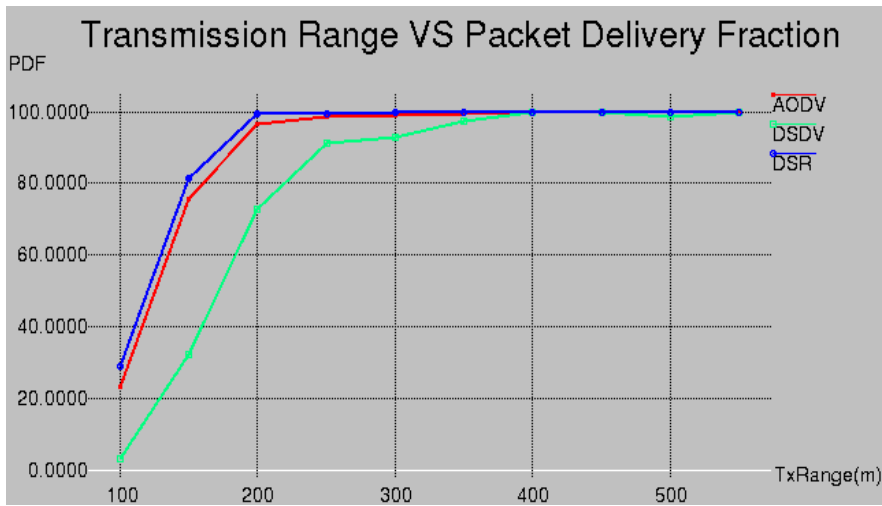

Figure 7, Transmission Range vs Packet Delivery Fraction

The following throughput graph shown in figure 8 looks exactly similar to the previous PDF graph. The only difference is the y axis metric. In the throughput graph, it is given in kbps. DSR provided good throughput if the Tx Range is below $350 \mathrm{~m}$. 


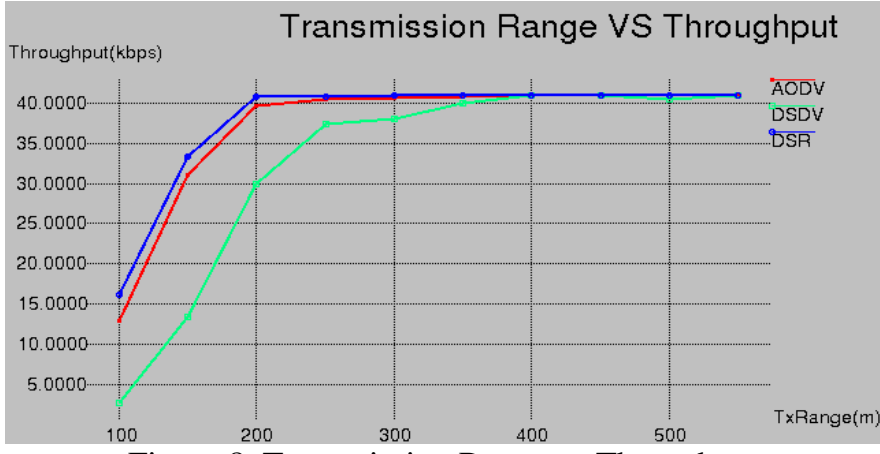

Figure 8, Transmission Range vs Throughput

\subsection{Observations and Findings}

As far as the experiments made on the topology under consideration, we can come to the following conclusions.

- All the routing algorithms performed almost equal while the transmission range of the nodes was half the length of the topological area. In other words, all the nodes in the network can be reached one or two hops from any other node then all the routing algorithms provided equal performance. We can understand this fact by closely observing the graphs after the T $x$ Range of 400 (half the length of topology).

- But even after the T $x$ Range of 400, the overhead and the total energy consumption were different in all the routing algorithms. But the performance with respect to all other metrics is same.

- All the compared algorithms provided entirely different level of performance with respect to all the considered metrics for up to the Tx Range of $200 \mathrm{~m}$. It means that, if the nodes are having the Tx Range below $200 \mathrm{~m}$, then the nodes can reach their destination only through multiple hops. So, routing through multiple hops obviously increases the end to end delay and overhead in routing. Further, the throughput as well as all other quality metrics gets affected considerably due to the multi hop routing process.

- In fact, a MANET routing algorithm can be classified as good or bad only based on its performance while the transmission ranges of the nodes were below the range of 350 or 400 meters

- If the Rx Range of the nodes were between $250 \mathrm{~m}$ to $350 \mathrm{~m}$, then the multi hop routing performance of all the nodes were considerably good and acceptable.

- So, the real differences in routing strategies of different protocols can be distinguishable only below the transmission range of 350 meters.

- And hence we can say that, any power aware routing protocol can really give good performance if it will dynamically change the Tx power to cover different ranges below 350 meters.

- The multi-path routing protocols were not energy efficient throughout all the range of transmissions.

- DSR is the overall good performer throughout all the transmission ranges.

- DSDV is the overall bad performer throughout all the transmission ranges and with respect to almost all the considered metrics.

\section{CONCLUSION AND FUTURE WORK}

We successfully evaluated the performance of routing protocols under different levels of transmission range/power of the nodes. Obviously the change in transmission range/power has a significant impact on the performance of the routing protocols.

The 'multi hop' routing protocols deliver acceptably good performance only at a particular levels of transmission ranges/powers. Even though the use of 'high' transmission rage/power will reduce lot of overheads and give excellent performance, we cannot use any arbitrarily high level of transmission power in most of the applications like sensor networks.

Even, minimizing the transmission range/power of the node will considerably increase the hops and so considerably increase the overhead in communication and reduce the overall performance.

Our results signifies that, any so called "good" power aware and energy efficient routing protocol should use different transmission ranges considerably very low T $x$ range performance (below the range of $400 \mathrm{~m}$ in this case) to get optimum level of performance. In a general sense, that "minimum" range is a very lower transmission range below the 'half' of the length of the whole topology. Then only it will give optimum performance in terms of power consumption as well as performance.

It means the power aware routing protocol should also be aware of the size of the topology under which it is functioning. Further, it should use that "topology length or breadth" information while making decisions in reducing or changing the transmission power of any individual nodes in the network. 
So, the future works address the way to estimate ways to set the transmission range/power of the nodes in a dynamic manner with respect to the several dynamic parameters of the network. Further, the dynamic size of the network topology and size plays very important role in deciding the maximum transmission range/power that a node can use during the dynamic MANET communication scenario.

Considering all the facts identified during this experiments, one can design a more efficient, power aware and topology aware routing protocol for MANET. Our future work will address these issues and propose a new power aware routing protocol.

\section{REFERENCES}

[1] J. Broch, D. A. Maltz, D. B. Johnson, Y.-C. Hu, and J. Jetcheva, "A Performance Comparison of MultiHop Wireless Ad Hoc Network Routing Protocols,” in Proceedings of MobiCOM'99, Dallas Texas, October 1998; also at http://www.monarch.cs.cmu.edu

[2] T. S. Rappaport, "Wireless Communications: Principles and Practice", 2nd ed., Prentice-Hall, Upper Saddle River, NJ, 2002.

[3] http://www.isi.edu/nsnam/ns/; NS-2 mobility extension from Rice Monarch, see http://www.monarch.cs.rice.edu/cmu-ns.html

[4] H. T. Friis, “A Note on a Simple Transmission Formula," Proc. IRE, Vol 34, no 5, pp.254-256, May 1946.

[5] C.Perkins and P.Bhagvat. "Highly dynamic destination sequenced distance-vector routing (DSDV) for mobile computers", ACM Computer Communications Review, pages 234-244, Oct 1994.

[6] R.K.Ahuja, T.L.Magnanti and J.B. Orlin. Network Flows, Theory, Algorithms and Applications. Prentice Hall, 1993

[7] D.B. Johnson and D.A.Maltz, "Dynamic source routing in ad hoc wireless networks": Mobile Computing, Kluwer Academic Publishers, Chapter 5, pp. 153-181,1996.

[8] D.A. Maltz, J. Broch, J. Jetcheva and D.B. Johnson, The effects of on-demand behavior in routing protocols for multi-hop wireless ad hoc networks, IEEE Journal on Selected Areas in Communications, August 1999.

[9] Sandeep Sahu, Sonam Jain "Geometric Routing Protocol based on Genetic Algorithm for Minimized Delay in MANETs" International Journal of Electronics Communication and Computer Technology (IJECCT) Volume 2 Issue 3, May 2012

[10] C. E. Perkins and E. M. Royer, "The Ad hoc On-Demand Distance Vector Protocol," in Ad hoc Networking, Addison-Wesley, 2000, pp. 173-219.

[11] C. E. Perkins, E. M. Belding-Royer, and S. Das, "Ad hoc On-Demand Distance Vector (AODV) Routing," RFC 3561, July 2003.

[12] Josh Broch, David B. Johnsson, David A. Maltz, The Dynamic Source Routing Protocol for Mobile Ad hoc networks. Internet Draft, draft-ietf-manet-dsr-00.txt, March 1998.

[13] Charles E. Perkins and Pravin Bhagwat,"Highly dynamic Destination-Sequenced Distance-Vector routing (DSDV) for mobile computers. In Proceedings of the SIGCOM '94 Conference on Communications Architecture, protocols and Applications, pages 234-244, August 1994.

[14] J. Broch, D.A. Maltz, D.B. Johnson, Y.C. Hu, and J. Jetcheva. A Performance Comparison of Multi-Hop Wireless Ad Hoc Network Routing Protocols. In Proceedings of the ACM/IEEE MobiCom, October 1998.

[15] W. Navidi and T. Camp, "Stationary distributions for the random waypoint mobility model," IEEE Transactions on Mobile Computing, vol. 3, no. 1, pp. 99-108, January-March 2004.

[16] S. Banerjee and A. Misra, "Energy efficient reliable communication for multi-hop wireless networks," Accepted for publication in Journal of Wireless Networks (WINET). 